\title{
Enfermedad de Hansen asociado a Síndrome de Reconstitución Inmune en Pacientes con VIH/SIDA
}

\section{Hansen disease associated with Immune Reconstitution Syndrome in Patients with HIV / AIDS}

Dra. Cristina Alarcón ${ }^{1}$, Dra. Aurelia Taboada ${ }^{2}$, Dr. Gustavo Benítez. Programa Nacional de Lucha contra el SIDA ${ }^{1}$ - Instituto de Medicina TropicaP

Introducción: El Instituto de Medicina Tropical es un centro de referencia para enfermedades tropicales e infecciosas de Paraguay. Se expone la coincidencia entre la infección por el Virus de la Inmunodeficiencia Humana (VIH) y su estado terminal el Síndrome de Inmunodeficiencia Adquirida (SIDA) con la lepra, pues ambas tienen en común un deterioro del sistema inmunitario y una misma ubicación geográfica. Se analiza esta coinfección en diferentes países y su evolución, no siendo más frecuente esta asociación que el VIH en los grupos control. Objetivo: El objetivo de este trabajo es comunicar nuestra experiencia del tratamiento de la lepra en pacientes con VIH/SIDA. Materiales y métodos. Mediante el análisis de las historias clínicas de los pacientes con diagnóstico de Lepra y VIH/SIDA, se evaluó el tratamiento $y$ el estado inmunológico. Resultados. A lo largo del periodo de estudio se trataron 6 pacientes con lepra $y$ VIH. De ellos el 66.6\% eran hombres, con el 33.3\% (2 pacientes) menores de 30 años, $50 \%$ de ellos Lepra Bordeline Lepromatosa y 50\% LB Tuberculoide. AI momento del estudio, 5 pacientes habían terminado el tratamiento, quedando uno con tratamiento hasta febrero del 2014 cumpliendo de manera regular. Conclusión. Se concluye que existe una asociación entre el estado inmunológico de los pacientes $y$ la del diagnóstico de Lepra. Pretendemos proseguir la vigilancia de ambas endemias en los próximos años.

Palabras clave: Enfermedad de Hansen, Lepra, IMT 
Abstract

Background: The Institute of Tropical Medicine is a reference center for tropical and infectious diseases of Paraguay. The coincidence between infection HIV (HIV) and terminally Acquired Immune Deficiency Syndrome (AIDS) is exposed to leprosy, as both have in common a deterioration of the immune system and a geographic location. We analyzed this coinfection in different countries and their evolution, being this association not more frequent than HIV association in the control groups. Objective:

The objective of this study was to report our experience in treating leprosy patients with HIV / AIDS. Materials and methods. Through the analysis of medical records of patients diagnosed with leprosy and HIV / AIDS, treatment and immune status was assessed.

Results. Throughout the study period, 6 patients with leprosy and HIV were discussed. Of these, $66.6 \%$ were men, $33.3 \%$ (2 patients) under $30,50 \%$ of them Borderline lepromatous leprosy and $50 \%$ LB Tuberculoid. At the time of the study, 5 patients had completed the treatment, leaving one treatment until February 2014 meeting regularly.

Conclution. We conclude that there is an association between the immune status of patients and the diagnosis of leprosy. We intend to continue monitoring both endemic in the coming years $T M I$

Key words: Hansen's disease, leprosy,

\section{Introducción}

El síndrome de inmunodeficiencia humana conocido por las siglas VIH-SIDA (1), y el mal de Hansen (2), son enfermedades estigmatizadas por la sociedad lo que se debe, fundamentalmente al impacto social producido por dichas entidades.

Desde la introducción de la terapia antirretroviral de alto impacto (HAART o TARGA) en el manejo del HIV, especialmente en regiones endémicas para la lepra, los pacientes coinfectados están desarrollando también lepra tuberculoide con lesiones activas. La lepra está siendo reportada en forma creciente como parte del síndrome inflamatorio de reconstitución inmune.

El SIRI generalmente afecta a individuos infectados con el virus de la inmunodeficiencia humana (VIH-1) en estadios avanzados de la enfermedad (recuento de linfocitos T CD4 $<200$ células $/ \mathrm{mm}^{3}$ ). Los signos clínicos de la inflamación aparecen con la asociación de infecciones oportunistas sobre todo por $M$. tuberculosis, CMV e infecciones herpéticas, cuando la terapia HAART ha logrado la reducción de la carga viral y un incremento en el número de linfocitos CD4 (3)

La asociación con enfermedad de Hansen ha sido poco descrita en la literatura mundial. Se ha reportado a la lepra como un ejemplo de infección relacionada con el SIRI, radicando su importancia en el reconocimiento de este síndrome en las áreas endémicas de esta enfermedad (4).

El curso clínico y la respuesta terapéutica al $M$. leprae en la co-infección con el VIH ha sido objeto de mucho debate (3). Con este trabajo queremos describir seis casos de SIRI desencadenado por la infección con $M$. leprae tras el inicio de la terapia TARGA con el objetivo de estudiar la asociación de ambas enfermedades en 
los pacientes que acudieron a consultorio externo del Programa Nacional de Lucha contra el SIDA (PRONASIDA) dependiente del Instituto de Medicina Tropical que es centro de referencia de Paraguay.

\section{Materiales y Métodos}

Diseño: Estudio retrospectivo, observacional, de corte transverso.

Sujetos de estudio: Pacientes adultos atendidos en el consultorio del PRONASIDA con diagnóstico de Enfermedad de Hansen y VIH desde enero de 2008 a diciembre 2013.

Para facilitar el reconocimiento y clasificación del SIRI asociado a lepra se ha sugerido la siguiente definición de caso:

1) Lepra o reacción leprosa dentro de los 6 a 12 meses de inicio de la terapia TARGA;

2) Bajo conteo de linfocitos CD4+ antes del inicio de la Enfermedad de Hansen;

3) Aumento en el conteo de linfocitos CD4+ luego del inicio de la Enfermedad de Hansen (modificado de Amerson)

Criterios de inclusión: Pacientes atendidos en el consultorio del PRONASIDA con diagnóstico de Enfermedad de Hansen y VIH desde enero de 2008 a diciembre 2013 con datos completos para la realización de este estudio.

Tamaño de muestra: Se notificaron un total de seis casos que reunieron los criterios de inclusión.

Métodos, Técnicas e Instrumentos para la recolección de datos
- Método: Investigación documental, fuente secundaria.

- Técnica: Revisión sistemática de fichas, según criterio de inclusión.

- Instrumento: Formulario impreso, elaborado en base a la ficha de consultorio de accidente laboral

\section{Plan de Tabulación y Análisis}

El procesamiento de los datos se llevó a cabo mediante el sistema Excel, las variables y sub-variables fueron expresados en valores absolutos y porcentaje, representado en gráficos.

\section{Procedimiento para le recolección de datos}

Se solicitó el permiso correspondiente por escrito al Director de la Institución para comunicar el fin de la investigación y su importancia, solicitando el acceso a las historias clínicas de los pacientes con Lepra y VIH.

Se procedió a codificar cada dato obtenido registrado en el formulario.

\section{Principios éticos}

Beneficencia: La investigación fue realizada con fines estrictamente académicos, por lo que además ayudará a aumentar el nivel de conocimiento, aclarar dudas e implementar acciones a nivel Institucional.

Se espera que con esta investigación se beneficie al sector estudiado y a la profesión, además de proporcionar información para nuevos emprendimientos y ser utilizado por las personas interesadas.

Principio de justicia: Se basa en la búsqueda del bien común y en igualdad de condiciones para todos los individuos que llegasen a ser partícipes de la investigación 
o su resultado, este principio vela por la imparcialidad en la distribución en los beneficios y riesgo.

Confidencialidad: Los datos obtenidos se mantuvieron en la máxima confidencialidad, que asegura el anonimato de los usuarios.

\section{Resultados}

1) Sexo femenino, WB 03.04.08 CD4: 211, CV: 13.376. ARV AZT/3TC NVP.

Piel: eritemas nodulares en miembros superiores e inferiores.

Diagnóstico: biopsia de piel.

Tratamiento. Talidomida $100 \mathrm{mgr} / \mathrm{d}$. Tipo: no tipificado

2) Sexo masculino, WB: 25.07.11, CD4: 274, CV: 6229, ARV: AZT/3TC + EFV.

Piel: eritemas violáceos nodulares en tórax anterior, muslo derecho, codo izquierdo, dorso y planta del pie izquierdo

Diagnóstico: Biopsia de piel.

Tratamiento: Dapsona + Rifampicina. Tipo: HBT.

3) Sexo femenino, WB 11.08.05 CD4: 277, CV: 6675. ARV: AZT/3TC + LOP/RIT

Piel: lesión levemente eritematosa con sensibilidad abolida en el centro, región del Tórax posterior.

Diagnóstico: Biopsia de piel.

Tratamiento: Dapsona + Rifampicina. Tipo: HBT

4) Sexo masculino, WB 06.05.11, CD4: 52, CV: 62916. ARV: D4T+ 3TC + EFV
Piel: lesiones violáceas en miembros superior e inferior.

Diagnóstico: Biopsia de piel.

Tratamiento. Dapsona +Rifampicina. Tipo HBT.

5) Sexo masculino. WB 02.02.11 CD4: 90, CV: 526305 ARV: AZT/3TC +LOP/RIT.

Piel: lesiones ovaladas múltiples violáceas en abdomen, miembros superiores e inferiores.

Diagnóstico: Biopsia de piel.

Tratamiento. Dapsona + Clofacimina. Tipo: HBT/HBB

6) Sexo masculino, WB: 10.07.08, CD4: 397, CV: 662, ARV: AZT/3TC + EFV.

Piel: eritemas violáceas nodulares en tórax anterior, muslo derecho, codo Izq., dorso y planta del pie izquierdo.

Diagnóstico: Biopsia de piel.

Tratamiento: Dapsona + Rifampicina. Tipo: HBT.

Hemos encontrado 6 pacientes con VIH que cursaron con diagnóstico de Enfermedad de Hansen y fueron atendidos en el consultorio del PRONASIDA.

Solo dos de los pacientes fue de sexo femenino (33\%). La edad comprendida fue de entre 29 y 50 , edad media de los pacientes fue de $38.5 \pm 5$.

En el grafico 1 se puede ver el histograma de edad de los pacientes. 


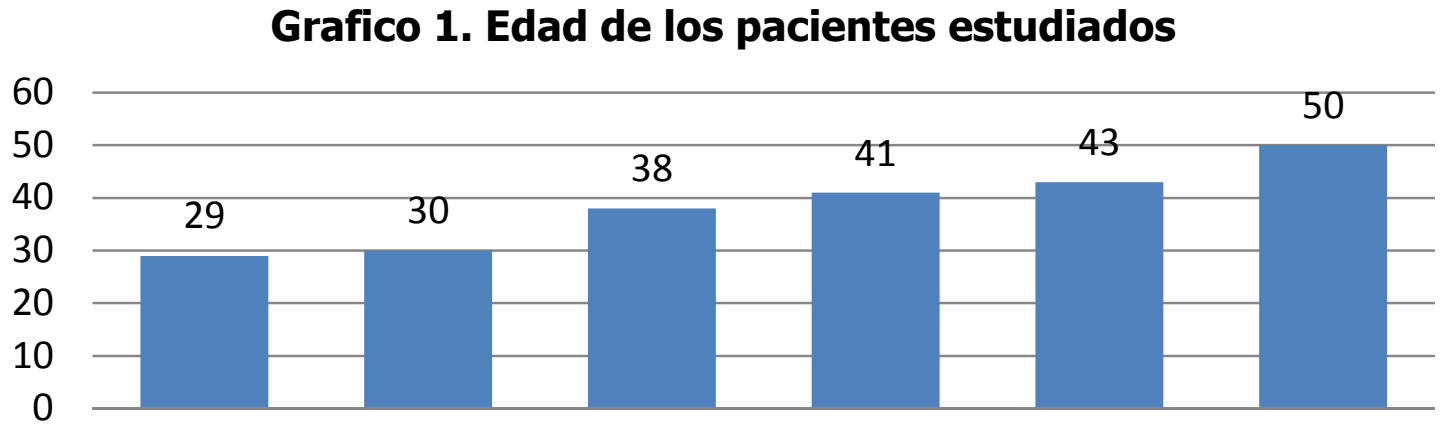

Paciente 1 Paciente 2 Paciente 3 Paciente 4 Paciente 5 Paciente 6

Lepra o reacción leprosa dentro de los 6 a 12 meses de inicio de la terapia TARGA
Los casos reportados se clasificaron en temprana o tardía según el inicio de la TARGA, encontrando así 3 pacientes (50\%) como tempranas y 3 pacientes con reacción lepromatosa tardía (Gráfico 2).

\section{Grafico 2. Clasificación de Lepra según el inicio de TARGA}

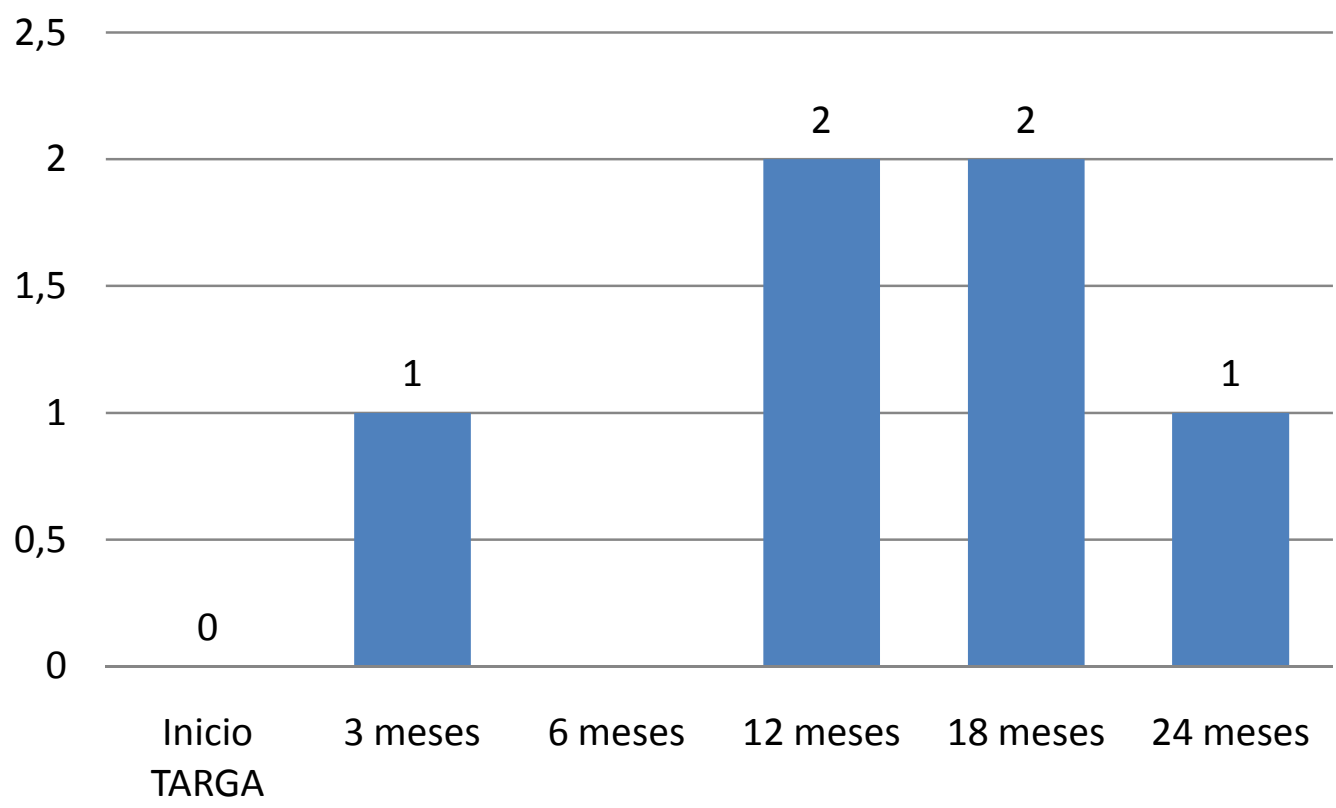

\section{Clasificación clínica de los pacientes según tratamiento}

De los 6 casos de lepra, 4 fueron tratados con rifampicina y dapsona supervisada cada 4 semanas y dapsona diaria durante 6 meses; mientras que 1 de los episodios de lepra fueron tratados con rifampicina y clofazimina y otro con dapsona y clofazimina, supervisado cada 4 semanas más clofazimina diario durante 12 meses, es así que los pacientes fueron clasificados como Lepra bordeline tuberculoide (4/6 $67 \%)$ y 1 paciente clasificado como HBT/HBB (bordeline Tuberculoide / bordeline - bordeline) $(1 / 6-17 \%)$, uno no fue clasificado

En este trabajo también pone de manifiesto la mortalidad nula en esta serie de casos debida a la lepra, ya que es una 
enfermedad crónica y es conocido que causa más morbilidad $(4,5)$.

\section{Discusión}

El Síndrome inflamatorio reconstitución inmune (SIRI), es un fenómeno bastante bien documentado pero poco entendido. El SIRI ocurre en el contexto de la restauración del sistema inmunológico debido al inicio de la terapia antirretroviral de alto impacto y se describe como una respuesta inmunológica alterada a un patógeno o antígeno previamente existente, que causa enfermedad clínica nueva o empeoramiento clínico de una enfermedad preexistente.

La emergencia de la pandemia del VIH/SIDA en los países tropicales en vías de desarrollo y la consecuente implementación de la terapia TARGA en estas regiones ha llevado a numerosos reportes de SIRI que ocurren asociados a infecciones tropicales (3).

En nuestra serie de casos, en el año 2008 encontramos los primeros casos de SIRI asociados a la lepra y desde entonces se han reportado otros 4 casos de esta asociación. La prevalencia del SIRI en estudios de cohorte en pacientes VIH positivos varía entre el 3 a más del $50 \%$ (57)

Al principio de la epidemia del VIH, se especuló que este llevaría a un aumento significativo en la prevalencia de la lepra en las regiones endémicas. Aunque la coinfección con VIH y Mycobacterium leprae se ha reportado en forma frecuente en regiones tropicales, la mayoría en Brasil y el África Sub-Sahariana, la epidemia del VIH parece no haber alterado substancialmente la epidemiología de la lepra en estas regiones. La infección por el VIH parece no aumentar en forma significativa el riesgo de infectarse con $M$. leprae, y la co-infección no predice un peor resultado. $(3,5)$

La presentación clínica de la enfermedad de Hansen depende de la respuesta del huésped, donde las lesiones cutáneas y neuropatía son comunes. En nuestro estudio, los 6 pacientes presentaron formas de lepra cutánea, de cualquier manera desde la introducción de la terapia con TARGA la forma HBT demostró predominio en nuestro reporte, como lo reportan estudios realizados en Brasil que predomina la forma bordeline tuberculoide. $(5,8-9)$.

Coincidentemente con los datos de la aparición de SIRI temprana y tardía que fueron $50 \%$ en ambos casos.

Los que tienen una mejor inmunidad celular tienden a presentar lesiones en la piel más asimétricas y pocos o ningún bacilo en el frotis de tejido. Esto ha sido referido como lepra tuberculoide. Por el contrario, la lepra lepromatosa se manifiesta típicamente como lesiones de piel generalizadas entre nódulos y placas con participación nerviosa más severa y se atribuye a aquellos con una menor respuesta inmune celular (5).

\section{Conclusión}

En cualquier paciente infectado por el VIH en tratamiento con TARGA que desarrolla placas eritematosas y trastornos de la sensibilidad, la lepra debe descartarse en primer lugar, sobre todo en área endémicas como nuestro país, por lo que los médicos deben estar conscientes de esta posibilidad, los pacientes presentan con frecuencia Lepra a causa del estado inmunológico deteriorado que facilita la infección por microorganismo oportunistas como el Mycobacterium sp. 
No obtendremos un buen control de la lepra mientras no prestemos más atención a los aspectos económicos y sociales de la sociedad donde prevalezca. Cualquier sistema de control de la lepra que ignore la falta de necesidades del enfermo como individuo estará condenado al fracaso (10).

Ante un paciente que inicia TARGA, además de la buena evaluación clínica se debe considerar al inicio de la reconstitución del CD4 con las lesiones en piel para llegar a un buen diagnóstico de lepra o a sus diagnósticos diferenciales.

En conclusión, la lepra continúa siendo un problema de salud pública en Paraguay. Queremos resaltar la importancia de los centros hospitalarios de tratamiento y control de la lepra, ya que realizan una importante labor en el tratamiento y manejo de las reacciones lepromatosas y por consiguiente en prevención y el tratamiento de las discapacidades causadas por la infección por el bacilo de Hansen. Es por ello, que la lepra debe seguir considerándose prioritaria y no deben disminuir los esfuerzos para mejorar la atención de los pacientes y reducir las incapacidades funcionales

\section{Referencias}

1. Quesada, E., E. Galban, A. Cádiz: Infección enfermedad por el virus de la Inmunodeficiencia Humana. La Habana: Editorial Científico-Técnica-37. 1987

2. Organización Mundial de la Salud: Epidemiología de la lepra en relación con la lucha antileprosa. Madrid: 1985 OMS 30-40 (impresión ligera).

3. Amerson $\mathrm{EH}$, Maurer TA. Immune reconstitution inflammatory syndrome and tropical dermatoses. Dermatol Clin 2011; 29: 39-43
4. Global AIDS response progress reporting 2013: Construction of core indicators for monitoring the 2011 UN Political Declaration on HIV/AIDS

5. Lockwood D, Lambert S. Human Immunodeficiency Virus and Leprosy: An Update. Dermatol Clin 2011; 29: 125128

6. Lawn S, Wood C, Lockwood D. Borderline Tuberculoid Leprosy: An Immune Reconstitution Phenomenon in a Human Immunodeficiency VirusInfected Person. Clin Infect Dis 2003; 36(1): e5-e6. doi: 10.1086/344446.

7. Pignataro $P$, Rocha Ada $S$, Nery $J A$, Miranda $A$, Sales AM, Ferreria $H$, et al. Leprosy and AIDS: Two cases of increasing inflammatory reactions at the start of highly active antiretroviral therapy. Eur J Clin Microbiol Infect Dis 2004; 23:408-11

8. Talhari C, Mira MT, Massone C, et al. Leprosy and HIV: a clinical, pathological, immunological and therapeutic study of a cohort from a Brazilian referral centre and infectious diseases. J Infect Dis 2010; 202 (3): 345-54.

9. Sarno EN, Illarramendi $X$, Nery JA, et al. HIV-M. leprae interaction: can HAART modify the course of leprosy? Public Health Rep 2008; 123: 206-212.

10. Latapí F. Los comandos del Centro Dermatológico Pascua nacieron en 1971 como idea del Dr. F. Latapí C. Comunicación personal 1972

Fecha de recepción: 16 de junio de 2014

Fecha de aceptación: 01 de diciembre de 2014

Solicitud de Sobretiros:

Dra. Cristina Alarcón

Servicio de Clínica Médica

Instituto de Medicina Tropical

gbenite@yahoo.com.ar 